\title{
Associations of lipoprotein particle profile and objectively measured physical activity and sedentary time in schoolchildren: a prospective cohort study
}

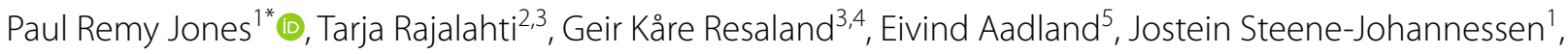
Sigmund Alfred Anderssen ${ }^{1,5}$, Tone Frost Bathen ${ }^{6}$, Trygve Andreassen ${ }^{7}$, Olav Martin Kvalheim² and Ulf Ekelund ${ }^{1}$

\begin{abstract}
Background: Our understanding of the mechanisms through which physical activity might benefit lipoprotein metabolism is inadequate. Here we characterise the continuous associations between physical activity of different intensities, sedentary time, and a comprehensive lipoprotein particle profile.
\end{abstract}

Methods: Our cohort included 762 fifth grade (mean [SD] age = 10.0 [0.3] y) Norwegian schoolchildren (49.6\% girls) measured on two separate occasions across one school year. We used targeted proton nuclear magnetic resonance $\left({ }^{1} \mathrm{H}\right.$ NMR) spectroscopy to produce 57 lipoprotein measures from fasted blood serum samples. The children wore accelerometers for seven consecutive days to record time spent in light-, moderate-, and vigorous-intensity physical activity, and sedentary time. We used separate multivariable linear regression models to analyse associations between the device-measured activity variables - modelled both prospectively (baseline value) and as change scores (followup minus baseline value) —and each lipoprotein measure at follow-up.

Results: Higher baseline levels of moderate-intensity and vigorous-intensity physical activity were associated with a favourable lipoprotein particle profile at follow-up. The strongest associations were with the larger subclasses of triglyceride-rich lipoproteins. Sedentary time was associated with an unfavourable lipoprotein particle profile, the pattern of associations being the inverse of those in the moderate-intensity and vigorous-intensity physical activity analyses. The associations with light-intensity physical activity were more modest; those of the change models were weak.

Conclusion: We provide evidence of a prospective association between time spent active or sedentary and lipoprotein metabolism in schoolchildren. Change in activity levels across the school year is of limited influence in our young, healthy cohort.

Trial registration: ClinicalTrials.gov, \#NCT02132494. Registered 7th April 2014

Keywords: Epidemiology, Lipoproteins, Metabolism, Physical activity, Sedentary time

*Correspondence: paulj@nih.no

1 Department of Sports Medicine, Norwegian School of Sport Sciences, Ullevål Stadion, Post Box 4014, 0806 Oslo, Norway

Full list of author information is available at the end of the article

\section{Background}

Cardiovascular diseases (CVDs) are the leading cause of death globally [1]. The associations between physical activity (PA), CVD incidence and mortality are wellestablished, and raising levels of PA is considered a cornerstone of disease prevention for both individuals original author(s) and the source, provide a link to the Creative Commons licence, and indicate if changes were made. The images or other third party material in this article are included in the article's Creative Commons licence, unless indicated otherwise in a credit line to the material. If material is not included in the article's Creative Commons licence and your intended use is not permitted by statutory regulation or exceeds the permitted use, you will need to obtain permission directly from the copyright holder. To view a copy of this licence, visit http://creativecommons.org/licenses/by/4.0/. The Creative Commons Public Domain Dedication waiver (http://creativeco mmons.org/publicdomain/zero/1.0/) applies to the data made available in this article, unless otherwise stated in a credit line to the data. 
and populations [2-4]. In children and adolescents, higher levels of PA are associated with better composite scores of cardiometabolic health $[5,6]$. However, the relationship with individual cardiometabolic risk factors, such as blood lipids, is inconsistent and most data are from cross-sectional studies [7]. It is also unclear whether these associations are independent of adiposity [8].

More detailed metabolic phenotyping-metabolomics-can improve our understanding of the mechanisms by which PA benefits metabolism by providing detailed information of the molecules and pathways involved [9]. A small number of studies have revealed associations of PA with a number of novel measures of lipid metabolism not observable with the standard lipid profile $[10,11]$. We have previously examined the theoretical effects of replacing time spent sedentary with moderate- to vigorous-intensity physical activity (MVPA) using isotemporal substitution, but were limited in our ability to examine temporality of the associations due to the cross-sectional design [12].

In this study, we used targeted proton nuclear magnetic resonance $\left({ }^{1} \mathrm{H}\right.$ NMR) spectroscopy to produce comprehensive lipoprotein particle profiles for our cohort of healthy schoolchildren, then examined the continuous associations with objectively measured PA of different intensities and sedentary time over one school year. We also explored potential confounding of associations by adiposity.

\section{Methods}

Additional information regarding blood sample handling and the ${ }^{1} \mathrm{H}$ NMR protocol are reported in the Supplementary Material.

\section{Sample population}

We drew our cohort from children who participated in the Active Smarter Kids (ASK) study; a cluster randomised controlled trial (RCT) in which the effect of a school-based PA intervention on academic performance was investigated (https://clinicaltrials.gov, \#NCT02132494) [13]. Of the 60 schools approached, 57 (1129 children) participated. The PA intervention was delivered over one academic year. Baseline testing took place in 2014. Changes in physical activity levels were of a similar degree for children who either received the intervention or did not [13]. We therefore pooled all children for this analysis.

\section{Ethics}

The Regional Committee for Medical Research Ethics approved the study protocol (2013/1893). Written consent was obtained from each child's parent(s) or legal guardian(s) and from school authorities prior to testing.
Procedures and methods abide by the World Medical Association's Declaration of Helsinki [14].

\section{Exposure variables}

The children wore triaxial accelerometers (ActiGraph GT3X+, ActiGraph LLC, Pensacola, FL) positioned on their right hip for seven consecutive days, but not during sleep or water-based activities. Children with at least four valid days of accelerometer measurements were included in the analytical sample. We considered a valid day $\geq 480$ min of monitor wear time between 0600 and 0000. This combination of valid days and minimum daily wear time per day has been shown to provide reliable estimates of physical activity measured by ActiGraph accelerometers in children [15]. Non-wear time was defined as $\geq 20 \mathrm{~min}$ of zero counts [16]. The accelerometer data were processed using commercially available KineSoft software (version 3.3.80, KineSoft, Loughborough, United Kingdom) and 10-s epochs. We classified PA intensity and sedentary time using the Evenson cut points of count data: sedentary time $\left(\leq 100\right.$ counts $\left.\mathrm{min}^{-1}\right)$, low-intensity physical activity (LPA; $>100$ and $<2296$ counts. $\mathrm{min}^{-1}$ ), moderate-intensity physical activity (MPA; $\geq 2296$ and $<4012$ counts $\cdot \mathrm{min}^{-1}$ ), and vigorous-intensity physical activity (VPA; $\geq 4012$ counts $\cdot \mathrm{min}^{-1}$ ) $[17,18]$.

\section{Outcome variables}

The children fasted overnight, and a trained nurse or phlebotomist drew blood serum samples between 0800 and 1000. NMR spectra were recorded on a Bruker Avance III $600 \mathrm{MHz}$ spectrometer (Bruker BioSpin $\mathrm{GmbH}$, Karlsruhe, Germany). We selected the lipoprotein NMR spectral regions quantitatively associated to lipoprotein concentrations as explanatory variables to partial least squares (PLS) modelling. The PLS model response variables were determined by high-performance liquid chromatography (HPLC) [19, 20]. In total, 106 serum samples were randomly selected for both HPLC and NMR analysis. We used a Monte Carlo resampling approach to calculate individual PLS models with optimal prediction ability for the HPLC data [21]. Lipoprotein particle numbers for all samples were predicted from these models, and the 20 lipoprotein subclasses were reduced to 15 [22]. Due to the elution of lipid-poor pre- $\beta_{1}$ high-density lipoproteins (HDLs), the "spherical particle model" for calculating particle number cannot be applied to the HDL7 minor subclass [20, 23]. Hence, the particle number of the HDL VS subclass in our study was calculated using HDL6 only. Lipoprotein measures available for subsequent analysis comprised: total serum cholesterol concentration; total triglycerides concentration; non-HDL cholesterol concentration (calculated by subtracting HDL cholesterol concentration from the total 
cholesterol concentration); particle number, cholesterol concentration and triglycerides concentration of 15 lipoprotein subclasses; and average particle diameter of very low-density lipoprotein (VLDL), low-density lipoprotein (LDL), and HDL particles. Though intact chylomicron particles cannot be distinguished from the largest VLDL particles using NMR spectroscopy, the nomenclature from the HPLC method, which does distinguish the two, was retained. For consistency, the chylomicron subclass was not incorporated when calculating measures of the VLDL class: VLDL cholesterol concentration, VLDL triglycerides concentration, or average VLDL particle size. However, given that the blood samples were drawn subsequent to an overnight fast, it is unlikely that the particles labelled chylomicrons are of intestinal origin and should therefore be considered very large VLDLs [24].

\section{Anthropometrics}

We measured body weight to the nearest $0.1 \mathrm{~kg}$ using an electronic scale (Seca 899, SECA GmbH, Hamburg, Germany). Height-with shoes removed, facing forwards-to the nearest $0.1 \mathrm{~cm}$ using a stadiometer (Seca 217, SECA $\mathrm{GmbH}$, Hamburg, Germany). We calculated body mass index (BMI) as weight divided by height squared $\left(\mathrm{kg} \cdot \mathrm{m}^{-}\right.$ ${ }^{2}$ ). Using a measuring tape (Seca 201, SECA GmbH, Hamburg, Germany), we took two measurements of waist circumference-between the lowest palpable rib and iliac crest, the child having gently exhaled-to the nearest $0.1 \mathrm{~cm}$. If the two measurements differed by more than $1.0 \mathrm{~cm}$ a third was taken; the mean of the two with the least difference was used for analysis. The proportions of overweight or obese girls and boys were calculated using the International Obesity Task Force's (IOTF) sex-specific BMI cut-offs, rounding down the children's ages at the time of testing to the nearest half-year [25].

\section{Sexual maturity}

Each child assessed their sexual maturity against a standard set of colour images and accompanying text descriptions that corresponded to the Tanner staging method [26]. The assessments took place in a private room and the children were accompanied by a researcher of the same sex to ensure their comfort. Low frequencies of children in Tanner categories 3,4 , and $5(n=66,5,2$, respectively of 1081 children with valid baseline data) were recorded and therefore combined into one category $(\geq 3)$.

\section{Educational attainment of parent(s) or guardian(s)}

This was considered as the highest level of attainment of a child's mother, father, or guardian, whichever was higher. Parent(s) or guardian(s) individually completed a custom self-report study questionnaire, selecting their level of educational attainment as one of six categories. Of the six, low frequencies were recorded in the lower four categories $(n=4,15,193,137$, for categories $1-4$, respectively of 1069 children with valid baseline data), so were combined into one category-Upper secondary school-for analysis.

\section{Statistical approach}

We examined the prospective associations between the mean daily time spent in different intensities of activity for each of the four activity variables (LPA, MPA, VPA, and sedentary time) measured at baseline and the 57 lipoprotein variables measured at follow-up using separate linear models. Each model was adjusted for sex and parent's/guardian's education, and the baseline values of the respective lipoprotein measure, mean daily accelerometer wear time, age, and sexual maturity. To examine the associations with change in mean daily time spent in different intensities of activity over the follow-up period, change scores were used (follow-up minus baseline). Accelerometer wear time was also modelled as a change score, and baseline values used for the other covariates. We repeated each analysis additionally including baseline waist circumference in the model to investigate potential confounding by adiposity. For each analysis, children with valid data for all model variables were included.

All activity and lipoprotein variables were converted to $z$-scores (mean $=0.0$; standard deviation $[\mathrm{SD}]=$ 1.0), hence the regression coefficients represent the $S D$ unit change in lipoprotein measure for a 1 SD increase in activity variable. Changes in mean daily time spent in different intensities of activity were modelled as the $z$-score of the change (follow-up minus baseline). To account for potential within-cluster correlation and to obviate the need to transform skewed outcome variables, cluster and heteroscedasticity robust standard errors were calculated, clustered on school. We used principal component analysis (PCA) to estimate the effective number of independent tests to use for multiple testing correction. The assumption of this approach is that the independence of principal components and degrees of freedom between the original lipoprotein measures are equivalent, and that dividing the alpha value by the number of principal components that explain $>95 \%$ of variance will produce only a small chance of false positives [27-29]. Using $z$-scores of the 57 lipoprotein measures, we calculated that the first five principal components explained $>95 \%$ of the variance. Hence, our Bonferroni-corrected threshold for assessing associations is $0.05 / 5=0.01$ (i.e., $p<0.01$ ). All analyses were conducted using $\mathrm{R}$ version 3.6.3 (R Foundation for Statistical Computing, Vienna, Austria). In addition to base $\mathrm{R}$ functions, we used a variety of packages within the tidyverse (1.3.0) suite for data manipulation. We performed the PCA analysis with factoextra 
(1.0.6) and the linear regression analysis using the estimatr (0.22.0) package, specifically the lm_robust() function. We plotted the results with ggplot2 (3.3.0) and the custom visualisation functions geom_stripes() and facet_col() available in the ggforestplot (0.0.2) and ggforce $(0.3 .1)$ packages, respectively.

\section{Results}

\section{Sample characteristics}

Our analytical sample for the prospective analyses comprised 762 children (49.6\% girls) with complete data and at least four valid days of accelerometer measurements (Fig. 1). There were 403 children (52.9\%) who had seven valid days of accelerometer data. Of these 762 children, 720 had at least four days of valid follow-up accelerometer data hence were included in the change score analyses. The average interval between baseline and follow-up accelerometer testing was 46.6 weeks. Correlations between the baseline and follow-up activity intensity measures in the change analysis were moderate (Pearson's $r=0.52$ for VPA, 0.53 for MPA, 0.57 for LPA, and 0.53 for sedentary time; $p<0.001$ for each).

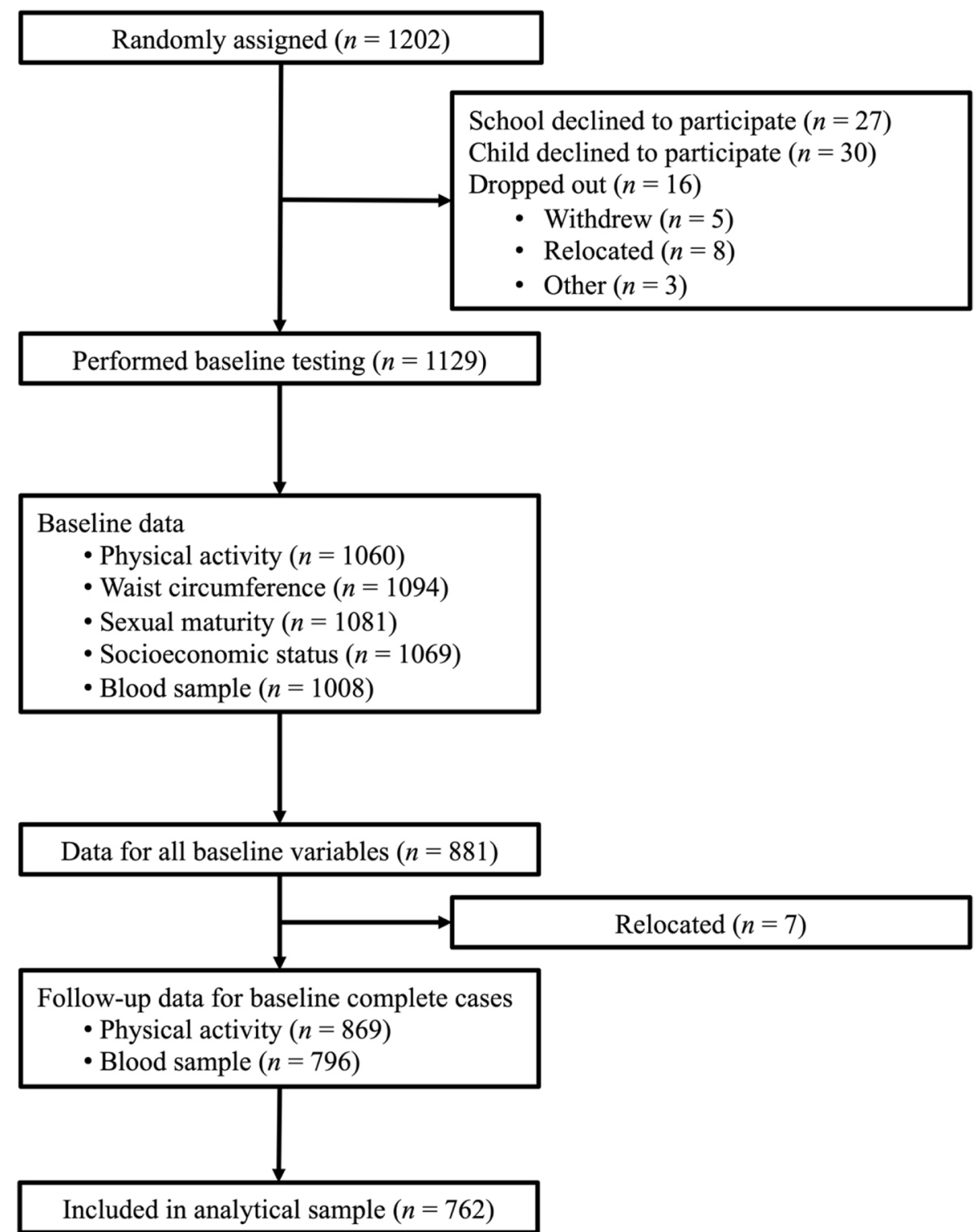

Fig. 1 Flow of participants through the study indicating number of children that had valid data available. The final analytical sample included those children that had valid data for all baseline variables and blood samples at follow-up 
Descriptive information for the analytical sample is given in Table 1. Means and SDs for the NMR lipoprotein measures are provided in Supplementary Material Table 1.

The 367 children not included in the prospective analyses tended to be slightly older and shorter (Supplementary Material Table 2).

\section{Vigorous-intensity physical activity}

In the prospective analysis, there were inverse associations between a 1 SD increment in VPA $\left(16.2 \mathrm{~min} \cdot \mathrm{d}^{-1}\right)$ and all measures of the VLDL particles (Fig. 2; Supplementary Material Table 3). For the individual measures of particle number, cholesterol concentration, and triglycerides concentration the effect sizes decreased from the largest to smallest of these particles (e.g., $-1.32 \mathrm{x}$ $10^{-1} \mathrm{nmol} \cdot \mathrm{L}^{-1}$ or $-0.13 \mathrm{SD} ; 95 \% \mathrm{CI}=-0.19,-0.06 ; p$ $<0.001$ for VLDL L1 particle number). The associations between VPA and all but one of the LDL measures were also inverse, though the effect sizes typically smaller than for the measures of larger ApoB-containing particles. The triglycerides concentrations of the two subclasses of the smallest LDL particles were an exception. For the HDL measures, the directions of associations tended to differ dependent on subclass. The effect sizes were mostly modest, though larger for the triglycerides concentrations of the two subclasses of the smallest HDL particles. The association with the average diameter of VLDL particles was inverse and effect size larger than those of the positive associations with the average diameter of LDL and HDL particles. Regarding the more traditional lipid measures, higher VPA was inversely associated with TC, non-HDL-C, and LDL-C concentrations, and positively associated with HDL-C concentration. The effect sizes were modest. In contrast, the effect size was larger for the inverse association with total triglycerides concentration. The pattern of associations remained broadly similar having included waist circumference as an additional covariate in the models. The degree of attenuation for individual measures ranged from small to moderate and tended to be greater for the subclasses of larger particles $(-8.60$ $\mathrm{x} 10^{-2} \mathrm{nmol} \cdot \mathrm{L}^{-1}$ or $-0.08 \mathrm{SD} ; 95 \% \mathrm{CI}=-0.14,-0.03 ; p$ $<0.01$ for VLDL L1 particle number).

The associations with a 1 SD change in VPA (14.9 min $\cdot \mathrm{d}^{-1}$ ) between baseline and follow-up measurement occasions were weak (Supplementary Material Fig. 1; Supplementary Material Table 7). Adjustment for waist circumference had a negligible effect on these associations.
Table 1 Characteristics of children included in the analytical sample

\begin{tabular}{|c|c|c|}
\hline Characteristic & $n(\%)$ & Mean (SD) \\
\hline Baseline & 762 & \\
\hline Age (years) & & $10.0(0.3)$ \\
\hline \multicolumn{3}{|l|}{ Sex } \\
\hline Girls & $378(49.6)$ & \\
\hline Boys & $384(50.4)$ & \\
\hline \multicolumn{3}{|l|}{ Anthropometry } \\
\hline Height (m) & & $143.1(6.7)$ \\
\hline Weight (kg) & & $37.2(8.1)$ \\
\hline BMI $\left(\mathrm{kg} \cdot \mathrm{m}^{-2}\right)$ & & $18.1(3.0)$ \\
\hline$\geq 25$ & $189(24.8)$ & \\
\hline$\geq 30$ & $40(5.2)$ & \\
\hline Waist circumference $(\mathrm{cm})$ & & $62.1(7.6)$ \\
\hline \multicolumn{3}{|l|}{ Parents' education } \\
\hline Upper secondary school & $241(31.6)$ & \\
\hline$<4$ years college/university & $229(30.1)$ & \\
\hline$\geq 4$ years college/university & $292(38.3)$ & \\
\hline \multicolumn{3}{|l|}{ Tanner stage } \\
\hline Stage 1 & $417(54.7)$ & \\
\hline Stage 2 & $297(39.0)$ & \\
\hline Stage $\geq 3$ & $48(6.3)$ & \\
\hline \multicolumn{3}{|l|}{ Physical activity } \\
\hline VPA $\left(\min \cdot d^{-1}\right)$ & & $32.2(16.2)$ \\
\hline $\operatorname{MPA}\left(\min \cdot d^{-1}\right)$ & & $45.1(12.8)$ \\
\hline LPA $\left(\min \cdot d^{-1}\right)$ & & $235.2(36.7)$ \\
\hline $\operatorname{SED}\left(\min \cdot d^{-1}\right)$ & & $466.5(57.5)$ \\
\hline MVPA $\geq 60\left(\mathrm{~min} \cdot \mathrm{d}^{-1}\right)$ & $563(73.9)$ & \\
\hline \multicolumn{3}{|l|}{ Lipid profilea } \\
\hline $\mathrm{TC}\left(\mathrm{mmol} \cdot \mathrm{L}^{-1}\right)$ & & $4.5(0.7)$ \\
\hline $\mathrm{LDL}-\mathrm{C}\left(\mathrm{mmo} \cdot \mathrm{L}^{-1}\right)$ & & $2.5(0.6)$ \\
\hline $\mathrm{HDL}-\mathrm{C}\left(\mathrm{mmol} \cdot \mathrm{L}^{-1}\right)$ & & $1.6(0.3)$ \\
\hline $\mathrm{TG}\left(\mathrm{mmol} \cdot \mathrm{L}^{-1}\right)^{\mathrm{b}}$ & & $0.7[0.5,0.9]$ \\
\hline Follow-up & 720 & \\
\hline \multicolumn{3}{|l|}{ Physical activity } \\
\hline$\Delta \mathrm{VPA}_{\mathrm{t} 2-\mathrm{t} 1}\left(\mathrm{~min} \cdot \mathrm{d}^{-1}\right)$ & & $-4.7(14.9)$ \\
\hline$\Delta \mathrm{MPA}_{\mathrm{t} 2-\mathrm{t} 1}\left(\min \cdot \mathrm{d}^{-1}\right)$ & & $-4.9(12.1)$ \\
\hline$\Delta \mathrm{LPA}_{\mathrm{t} 2-\mathrm{t} 1}\left(\mathrm{~min} \cdot \mathrm{d}^{-1}\right)$ & & $-14.3(33.3)$ \\
\hline$\Delta \mathrm{SED}_{\mathrm{t} 2-\mathrm{t} 1}\left(\mathrm{~min} \cdot \mathrm{d}^{-1}\right)$ & & $28.3(52.8)$ \\
\hline MVPA $\geq 60\left(\mathrm{~min} \cdot \mathrm{d}^{-1}\right)$ & $451(62.6)$ & \\
\hline \multicolumn{3}{|l|}{ Lipid profile ${ }^{a}$} \\
\hline $\mathrm{TC}\left(\mathrm{mmol} \cdot \mathrm{L}^{-1}\right)$ & & $4.5(0.6)$ \\
\hline $\mathrm{LDL}-\mathrm{C}\left(\mathrm{mmol} \cdot \mathrm{L}^{-1}\right)$ & & $2.6(0.6)$ \\
\hline $\mathrm{HDL}-\mathrm{C}\left(\mathrm{mmol} \cdot \mathrm{L}^{-1}\right)$ & & $1.6(0.3)$ \\
\hline $\mathrm{TG}\left(\mathrm{mmo} \cdot \mathrm{L}^{-1}\right)^{\mathrm{b}}$ & & $0.6[0.5,0.9]$ \\
\hline
\end{tabular}

Abbreviations: BMI body mass index, HDL-C high-density lipoprotein cholesterol, IQR interquartile range, $L D L-C$ low-density lipoprotein cholesterol, $L P A$ light-intensity physical activity, MPA moderate-intensity physical activity, MVPA moderate- to vigorous-intensity physical activity, SD standard deviation, SED sedentary time, TC total cholesterol, TG triglycerides, VPA vigorous-intensity physical activity

${ }^{a}$ Measured using clinical chemistry. LDL-C estimated using the Friedewald formula

${ }^{\mathrm{b}}$ Median [IQR] 


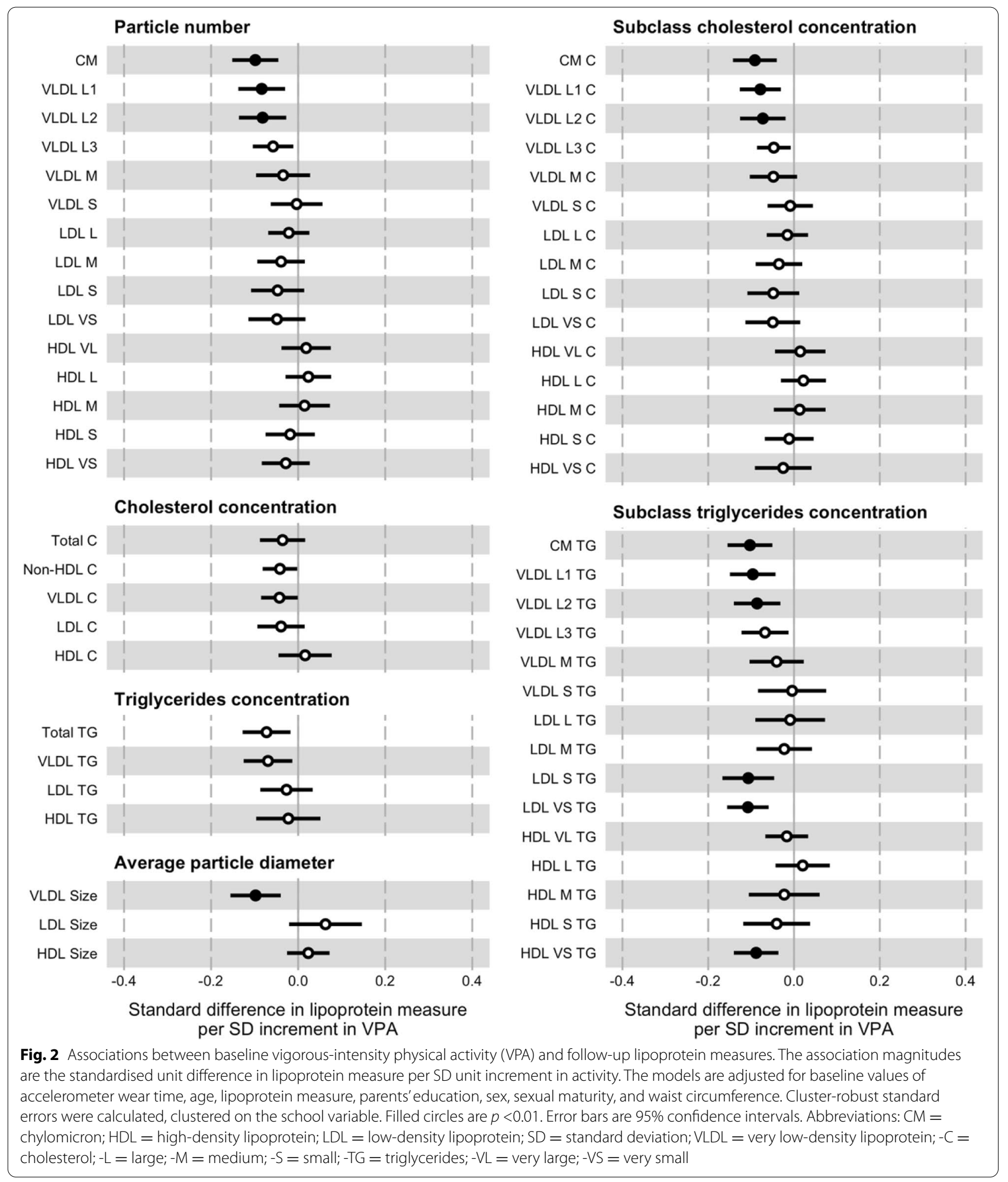




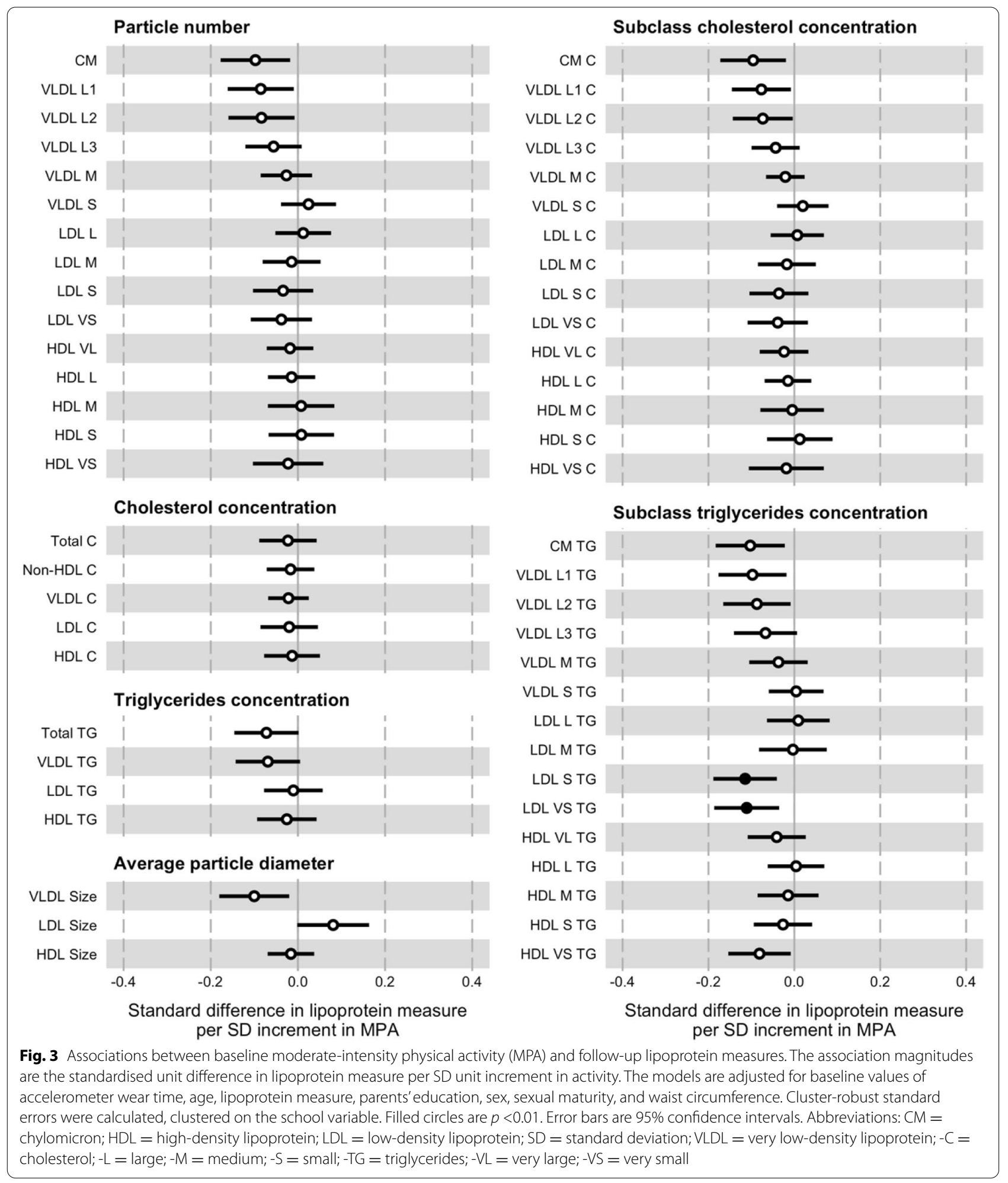

\section{Moderate-intensity physical activity}

In the prospective analysis, the pattern of associations between a $1 \mathrm{SD}$ increment in MPA $\left(12.8 \mathrm{~min} \cdot \mathrm{d}^{-1}\right)$ and the lipoprotein measures was broadly similar to that of a 1 SD increment in VPA (Fig. 3; Supplementary Material Table 4). The effect sizes were smaller for all but four of 
the 57 individual measures (e.g., $-1.14 \times 10^{-1} \mathrm{nmol} \cdot \mathrm{L}^{-1}$ or $-0.11 \mathrm{SD}$; $95 \% \mathrm{CI}=-0.19,-0.03 ; p<0.01$ for VLDL L1 particle number). Many of the coefficients were close to null. Including waist circumference as an additional covariate in the models had a negligible effect.

Generally, the pattern of associations with a $1 \mathrm{SD}$ change in MPA (12.1 $\left.\mathrm{min} \cdot \mathrm{d}^{-1}\right)$ was similar to those in the VPA change model, though the effect sizes for many individual measures were larger (Supplementary Material Fig. 2; Supplementary Material Table 8). Adjustment for waist circumference had a negligible effect on these associations.

\section{Light-intensity physical activity}

In contrast to the VPA and MPA analyses, the associations between a $1 \mathrm{SD}$ increment in LPA $\left(36.7 \mathrm{~min} \cdot \mathrm{d}^{-1}\right)$ and the VLDL measures tended to be more modest (Fig. 4; Supplementary Material Table 5). The effect sizes with certain individual subclasses were small, and those with the VLDL L3 and VLDL M subclasses almost null (e.g., $-4.53 \times 10^{-2} \mathrm{nmol} \cdot \mathrm{L}^{-1}$ or $-0.04 \mathrm{SD}$; $95 \% \mathrm{CI}=$ $-0.12,0.03 ; p=0.24$ for VLDL L1 particle number). In contrast to the VPA and MPA analyses, the directions of associations with the LDL subclass measures tended to be positive, though the distinct inverse associations with the triglycerides concentrations of the LDL S and LDL VS subclasses were replicated. The divergent directions of associations between the particle numbers and cholesterol concentrations of the HDL subclasses were apparent, but in the opposite directions to those in the VPA analysis. The effect sizes for these measures tended to be larger than in the VPA analysis, though not for the subclass triglycerides concentrations. Including waist circumference as an additional covariate in the models had a limited effect.

The associations between a 1 SD change in LPA (33.3 $\min \cdot \mathrm{d}^{-1}$ ) and the lipoprotein particle profile tended to be weak (Supplementary Material Fig. 3; Supplementary Material Table 9). Adjustment for waist circumference had a negligible effect on these associations.

\section{Sedentary time}

The associations between a 1 SD increment in sedentary time $\left(57.5 \mathrm{~min} \cdot \mathrm{d}^{-1}\right)$ were typically stronger with the VLDL particle measures (Fig. 5; Supplementary Material Table 6). The directions of associations with all but one of these measures were the opposite of those in the prospective analysis of MPA and all but the VLDL S subclass in the VPA analysis (e.g., $1.22 \times 10^{-1} \mathrm{nmol} \cdot \mathrm{L}^{-1}$ or $0.12 \mathrm{SD}$; $\mathrm{CI}=0.03,0.21 ; p=0.01$ for VLDL L1 particle number). Though the effect sizes were smaller, the pattern of effect sizes decreasing from the largest to the smallest particles was replicated. For the HDL subclasses, the directions of associations with measures of particle numbers and cholesterol concentrations tended to differ dependent on the particle size, though the majority of individual effects were small to medium. The effect sizes for the triglycerides concentrations of the two subclasses of the smallest LDL particles were again more pronounced compared to other LDL subclass measures, which were typically small to null. The effect sizes of the associations for the triglycerides concentrations of the subclasses of the largest and smallest HDL particles were relatively large compared to the other HDL subclasses. The degree of attenuation for individual measures having included waist circumference in the model were generally small.

The directions of association between a 1 SD change in sedentary time $\left(52.8 \mathrm{~min} \cdot \mathrm{d}^{-1}\right)$ and the lipoprotein measures tended to be the opposite of those in the VPA and MPA change models, and the effect sizes smaller (Supplementary Material Fig. 4; Supplementary Material Table 10). Adjustment for waist circumference had a negligible effect on these associations.

\section{Discussion}

We aimed to investigate the prospective associations of objectively measured PA and sedentary time with a comprehensive lipoprotein particle profile across the school year in fifth grade schoolchildren. We found that, broadly speaking, higher levels and higher intensities of PA are associated with an apparently favourable profile, whereas greater time spent sedentary seems detrimental. Effect sizes were modest, however, which suggests that the influence of PA on individual measures is limited. Associations with changes in PA or sedentary time were weak, which may potentially be due to the small overall changes in this active population.

Previous studies have reported similar beneficial lipoprotein particle profiles with higher levels of PA. In a study of device-measured physical activity and a comprehensive metabolic profile in adolescents, the authors showed a number of beneficial associations with MVPA, such as inverse associations with the triglycerides concentrations of LDL and VLDL subclasses, that were comparatively more robust than and tended to be in the opposite direction to those with sedentary time [10]. Associations between the metabolic profile and 3-year change in activity were generally weak. In another study, metabolic profiles of adults who self-reported their level of PA as "active" were compared to those who reported being "inactive" on two occasions at least five years apart [11]. Being consistently active was inversely associated with the particle concentrations of all ApoB-containing lipoprotein subclasses, a number of measures of subclass triglycerides concentration, and positively associated with both the particle concentrations of and cholesterol 


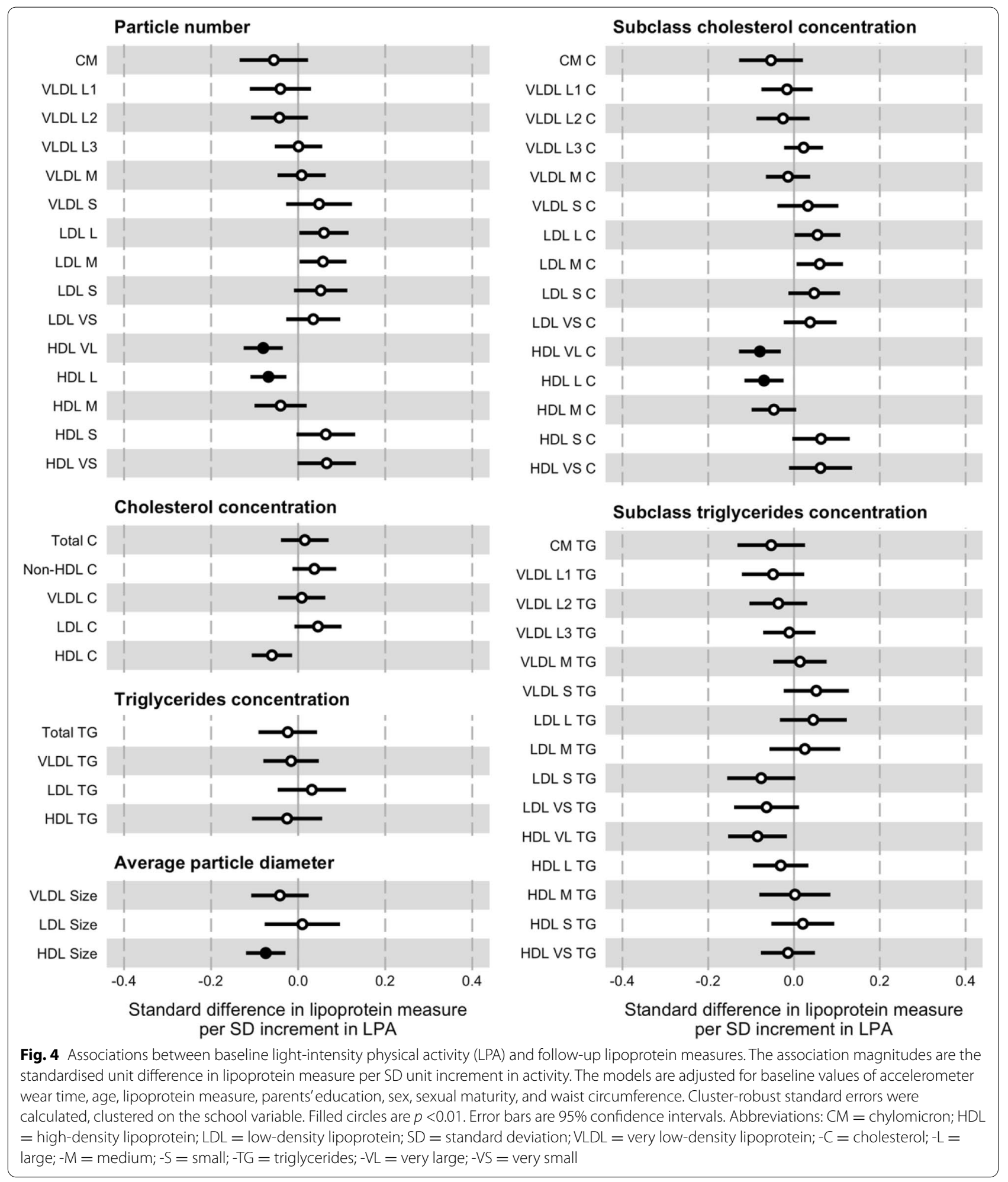

concentrations of the larger HDL subclasses. Many of the associations were more pronounced compared to those in our results, which could be for several reasons.
Firstly, the effect sizes reported for each lipoprotein measure were expressed using the SD unit difference in leisure-time PA between those categorised as active or 


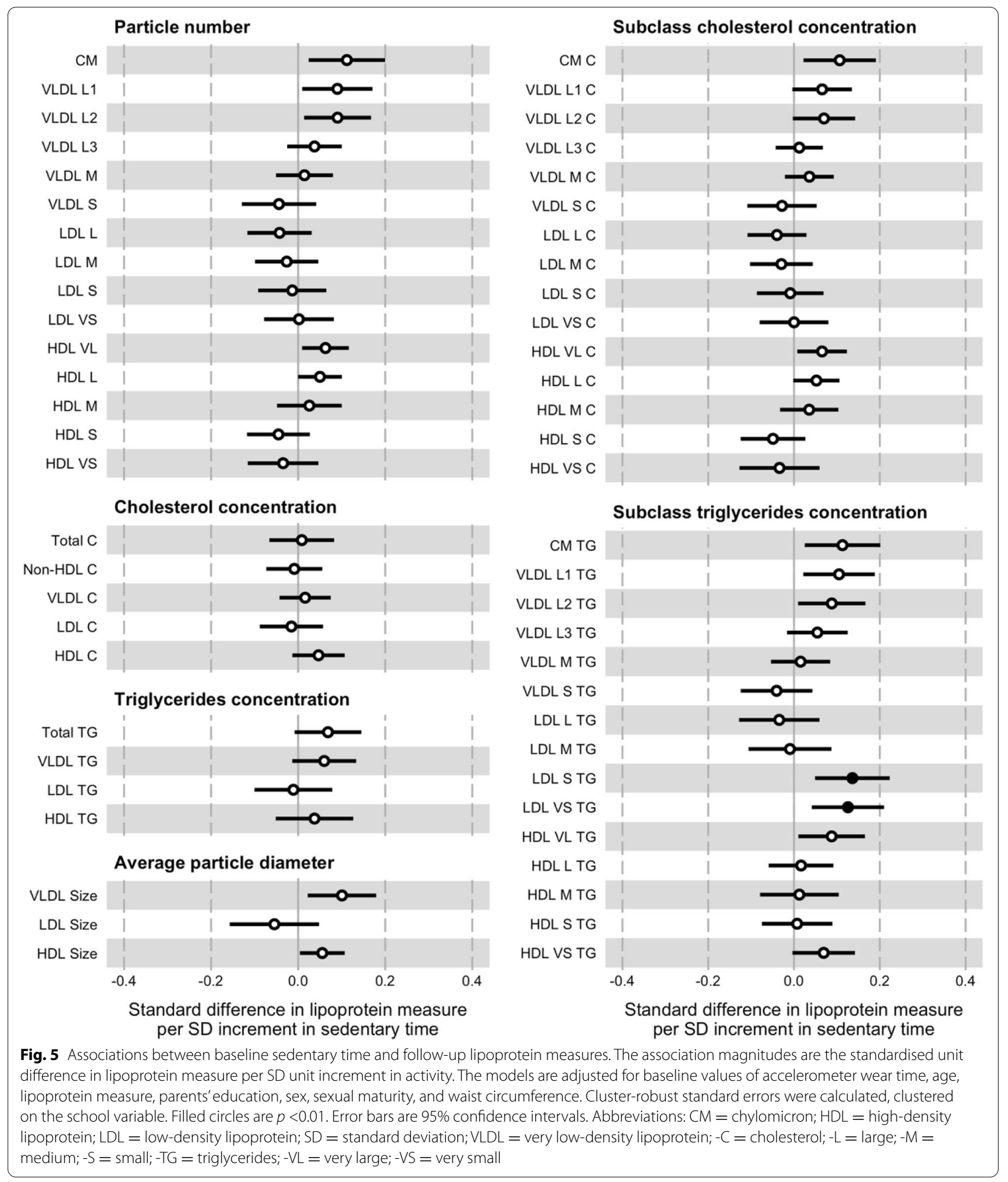

inactive, which are likely to be greater than the SD unit increments of the continuous PA intensity measures used in our study. The participants tended to be older and the time elapsed between the baseline and follow-up measurements far greater (e.g., 16 years in one of the included cohorts) than in our study. Thus, there is more likely to 
be greater variance in metabolism due to the accumulation of comorbidities with time and age. Also, activity levels of the included participants were likely more consistent across the two time points given that consistent level of activity was a criterion for inclusion.

Recent evidence suggests that the well-recognised causal effect of LDL on atherosclerotic CVD risk may not be a result of the lipid mass that the particles carry, but of the concentration of particles in the circulation [30-32]. Apolipoprotein $\mathrm{B}$ is the primary apolipoprotein of chylomicron, VLDL, and LDL particles, and likely the causal trait that enables the lipids carried by these lipoproteins to exert their influence on CVD risk [33, 34]. Moreover, all ApoB-containing lipoproteins up to $70 \mathrm{~nm}$ diameter, which includes triglyceride-rich and cholesterol-rich chylomicron and VLDL remnant particles, can penetrate the arterial intima and are thought to be similarly atherogenic [35]. Our results demonstrate relatively stronger associations of PA and sedentary time with VLDL particles, compared to the LDLs. Given that the number of circulating particles likely determines the probability of them entering and being retained in the intima, any cardioprotective effects of increased PA or reduced time spent sedentary seem likely expressed through the metabolism of these larger apolipoprotein B-containing subclasses [36]. Unexpectedly, LPA was positively associated with many LDL subclass measures. This contradicts existing literature, which has shown that LPA has minimal effect on LDL-C concentration and is possibly due to confounding whereby those children who spend more time in LPA spend less time in MPA or VPA [37].

Given that VLDLs are the primary carriers of triglycerides and triglycerides the primary lipid component of VLDLs, any effect of PA on VLDL particles will therefore likely be due to an effect on triglycerides metabolism. Though VLDL triglycerides appear not to play a significant role as substrate for lipid oxidation during exercise, increased rates of VLDL triglycerides clearance subsequent to a bout of aerobic exercise have been demonstrated [38, 39]. This exercise-induced reduction in circulating triglycerides concentration is typically abolished within 48 hours after the exercise bout [40]. Thus, the prospective associations of VPA and MPA with lower levels of VLDL measures in this study may reflect the acute effects of consistent repeated exercise-induced reductions in triglycerides concentration across the follow-up period, as opposed to more permanent metabolic adaptations to chronic PA behaviour. This finding supports that of the aforementioned study of adolescents in which the cross-sectional associations between objectively measured MVPA and the metabolic profile did not differ based on previous activity levels, which the authors interpreted as the effect of PA being dependent on recent engagement and consequently reversible if activity ceases [10].

Substantial structural, compositional, and functional heterogeneity exists between HDL particles. In addition to reverse cholesterol transport, HDL particles participate in antioxidative, anti-inflammatory, and anti-infectious activities, among others, and this assortment of biological functions seems to be mediated by different particle subpopulations [41]. Surprisingly, in our study particle numbers and cholesterol concentrations of the HDL subclasses were directionally consistent between VPA and sedentary time, suggesting a shift towards larger HDL particles and increased serum HDL cholesterol. However, the associations were weak in both analyses and negligible in the MPA model, so may not be true effects. If true, it is challenging to provide a mechanistic explanation for this apparent paradox contingent on energy expenditure alone, especially given that the associations were in the reverse direction and quite robust in the LPA analyses. Instead, these results may reflect the poor characterisation by particle number or lipid mass of HDL physicochemical and functional heterogeneity, and our incomplete understanding of how HDL function changes with either total PA or PA of different intensities. Historically, that higher HDL cholesterol levels are associated with higher levels of PA and lower CVD indicated a potential means through which PA exerts its cardioprotective effect [42]. However, recent evidence from Mendelian randomisation and clinical trials in which HDL cholesterol concentration was increased significantly with pharmacotherapy but failed to result in a concomitant reduction in CVD event rate compared to placebo indicate that a direct causal effect of HDL cholesterol level on CVD is unlikely [43-45]. Furthermore, there is preliminary evidence that exercise benefits some HDL attributes independent of changes in HDL cholesterol [46]. Consequently, greater research effort has been directed to quantifying HDL functionality, its influence on CVD risk, and the effects of PA on HDL beyond the traditional lipid profile [47, 48]. The generally modest associations with HDL measures in our results suggest the cardioprotective effects of PA are either inadequately characterised by measures of particle number and lipid load, or alternatively, indicative of limited metabolic perturbation in our young, healthy cohort.

In our analyses, associations were moderately attenuated having adjusted for waist circumference, which suggests an independent effect of PA and sedentary time on our lipoprotein measures. Adiposity has been shown to be causally associated with the lipoprotein particle profile in young adults, and that it mediates a proportion of the beneficial effects of MVPA on lipid measures $[8,49]$. There is also robust evidence that higher levels 
of adiposity are causal for lower total PA, MVPA, and increased time spent sedentary in children, and these associations are much stronger when adiposity is modelled as the exposure rather than the outcome [50]. Considered in the context of these studies, our results suggest that increasing VPA or MPA could benefit the lipoprotein particle profile, but an intervention that achieves a concomitant reduction in, or prevents increases in, adiposity would likely be synergistic. Furthermore, since the effects of PA on lipoprotein metabolism are likely acute and not maintained beyond a few days, any preventative programme would require frequent, repeated engagement in PA maintained across the life course. However, given that our study is observational, discussion of designing interventions is hypothetical. Replication of our findings in larger, more diverse populations with longer follow-up and greater metabolic variance are required to determine whether the potentially protective effects of PA are causal and what their impact on health might be.

\section{Strengths and limitations}

The use of a targeted metabolomics platform enabled us to investigate a variety of lipoprotein measures, providing a more nuanced description of the associations with PA and sedentary time than could be achieved with a standard lipid profile. We modelled the activity measures both prospectively and as change scores, adjusting for the baseline value of each respective lipoprotein measure, which enabled us to examine the temporality of associations. The blood samples were drawn with the children having fasted and at a consistent time of day, limiting potential variability due to dietary intake and daily activity. We had a high level of participant compliance with the PA assessment, though we acknowledge that one week of PA assessed at two time points may not be fully representative of behavioural variability over many months. Furthermore, substantial intraindividual variation has been reported when measuring children's PA using accelerometers over a 1-year period such that the true regression coefficients may be underestimated by up to 50\% [51]. Device-based measures of PA are less prone to the biases typical of self-report activity data, such as participant exaggeration.

There were also several limitations. Our data are observational hence we cannot exclude unmeasured confounding from biasing our effect estimates. Importantly, we did not have dietary information for our children. Nonfasted samples are considered more representative of the predominant metabolic state [24]. Our use of fasted samples precluded us from investigating the potential for PA to mitigate the postprandial rise in triglycerides and commensurate rise in circulating chylomicrons, which are thought to contribute to increased atherosclerotic risk [52]. The children that participated in the ASK study were young and the period over which they were followed short, so metabolic variance is likely limited. It would be instructive to follow them for a longer period or as they transition through adolescence into adulthood to observe whether the potentially beneficial effects of PA augment with age. Our cohort are homogenous and also highly active in comparison to the other adolescents both within Norway and globally, which likely limits the generalisability of our findings to other populations [53]. Lastly, we modelled each activity variable separately and are therefore unable to assess their independent associations with the lipoprotein particle profile.

\section{Conclusion}

Our study shows that more time spent being physically active-especially at higher intensities-is prospectively associated with a favourable lipoprotein particle profile, whereas more time spent sedentary appears to be detrimental. These associations are largely independent of adiposity. If causal, the mechanisms that drive these benefits are likely due to alterations in triglycerides metabolism, which may explain the typically inconclusive results of PA studies that only examine the standard lipid profile.

\begin{abstract}
Abbreviations
${ }^{1}$ H NMR: Proton nuclear magnetic resonance; ASK: Active Smarter Kids; BMI: Body mass index; CVD: Cardiovascular disease; HDL: High-density lipoprotein; HPLC: High-performance liquid chromatography; IOTF: International Obesity Task Force; LDL: Low-density lipoprotein; LPA: Low-intensity physical activity; MPA: Moderate-intensity physical activity; MVPA: Moderate- to vigorous-intensity physical activity; PA: Physical activity; PCA: Principal component analysis; PLS: Partial least squares; RCT: Randomised controlled trial; SD: Standard deviation; VLDL: Very low-density lipoprotein; VPA: Vigorous-intensity physical activity.
\end{abstract}

\section{Supplementary Information}

The online version contains supplementary material available at https://doi. org/10.1186/s12966-022-01244-w.

\section{Additional file 1.}

\section{Acknowledgements}

We are grateful to the children, parents and guardians, teachers, and principals from the participating elementary schools for their time, effort and cooperation. We thank our colleagues and students at the Western Norway University of Applied Sciences (formerly Sogn og Fjordane University College) for their assistance during the data collection. We also thank the Førde Central Hospital staff who coordinated the collection of blood samples. The NMR experiments were performed at the MR Core Facility, NTNU — Norwegian University of Science and Technology.

\section{Authors' contributions}

PRJ, GKR, EA, JS-J, SAA, OMK, and UE contributed to the conception and design of the work. TR, GKR, EA, TFB, and TA contributed to data acquisition. PRJ, TR, TFB, TA, and OMK contributed to data analysis. PRJ, EA, OMK, and UE contributed to interpretation of the results. PRJ and UE drafted the manuscript. All authors critically revised the manuscript, gave final approval, and 
agree to be accountable for all aspects of the work ensuring integrity and accuracy.

\section{Funding}

PRJ's fellowship position is funded by a Research Council of Norway grant awarded to UE as part of its Toppforsk programme [Generation Sedentary; \#249932/F20]. The ASK study was supported by the Research Council of Norway [\#221047/F40]; and the Gjensidige Foundation [\#1042294]. TR received funding from Førde Health Trust to perform the NMR lipoprotein analysis. The MR Core Facility is funded by the Faculty of Medicine, NTNU — Norwegian University of Science and Technology, and Central Norway Regional Health Authority (Helse Midt-Norge RHF). The study funders had no role in the design of the study; data collection, analysis, or interpretation; writing the report; or the decision to submit the paper for publication. The corresponding author had full access to all the study data and had final responsibility for the decision to submit for publication.

\section{Availability of data and materials}

The datasets used and/or analysed during the current study are available from the corresponding author on reasonable request.

\section{Declarations}

\section{Ethics and consent to participate}

The Regional Committee for Medical Research Ethics approved the study protocol. Written consent was obtained from each child's parent(s) or legal guardian(s) and from school authorities prior to testing.

\section{Consent for publication}

Not applicable

\section{Competing interests}

The authors declare that there are no competing interests.

\section{Author details}

'Department of Sports Medicine, Norwegian School of Sport Sciences, Ullevål Stadion, Post Box 4014, 0806 Oslo, Norway. ${ }^{2}$ Department of Chemistry, University of Bergen, Bergen, Norway. ${ }^{3}$ Førde Health Trust, Førde, Norway. ${ }^{4}$ Center for Physically Active Learning, Faculty of Education, Arts and Sports, Campus Sogndal, Western Norway University of Applied Sciences, Sogndal, Norway. ${ }^{5}$ Department of Sport, Food and Natural Sciences, Western Norway University of Applied Sciences, Sogndal, Norway. ${ }^{6}$ Department of Circulation and Medical Imaging, NTNU — Norwegian University of Science and Technology, Trondheim, Norway. ${ }^{7}$ MR Core Facility, Department of Circulation and Medical Imaging, NTNU — Norwegian University of Science and Technology, Trondheim, Norway.

Received: 9 June 2021 Accepted: 16 December 2021

Published online: 21 January 2022

\section{References}

1. Roth GA, Abate D, Abate KH, Abay SM, Abbafati C, Abbasi N, et al. Global, regional, and national age-sex-specific mortality for 282 causes of death in 195 countries and territories, 1980-2017: a systematic analysis for the Global Burden of Disease Study 2017. Lancet. 2018;392(10159):1736-88.

2. Kraus WE, Powell KE, Haskell WL, Janz KF, Campbell WW, Jakicic JM, et al. Physical activity, all-cause and cardiovascular mortality, and cardiovascular disease. Med Sci Sports Exerc. 2019;51(6):1270-81.

3. Arnett DK, Blumenthal RS, Albert MA, Buroker AB, Goldberger ZD, Hahn EJ, et al. 2019 ACC/AHA Guideline on the Primary Prevention of Cardiovascular Disease: a report of the American College of Cardiology/ American Heart Association Task Force on Clinical Practice Guidelines. Circulation. 2019;140(11):e596-646.

4. World Health Organization. Guidelines on physical activity and sedentary behaviour. Geneva: World Health Organization; 2020.

5. Cristi-Montero C, Chillón P, Labayen I, Casajus JA, Gonzalez-Gross M, Vanhelst J, et al. Cardiometabolic risk through an integrative classification combining physical activity and sedentary behavior in European adolescents: HELENA study. J Sport Health Sci. 2019;8(1):55-62.

6. Tarp J, Child A, White T, Westgate K, Bugge A, Grøntved A, et al. Physical activity intensity, bout-duration, and cardiometabolic risk markers in children and adolescents. Int J Obes. 2018;42(9):1639-50.

7. Poitras VJ, Gray CE, Borghese MM, Carson V, Chaput J-P, Janssen I, et al. Systematic review of the relationships between objectively measured physical activity and health indicators in school-aged children and youth. Appl Physiol Nutr Metab. 2016;41 (6 (Suppl. 3)):S197-239.

8. Tarp J, Bugge A, Andersen LB, Sardinha LB, Ekelund U, Brage S, et al. Does adiposity mediate the relationship between physical activity and biological risk factors in youth?: a cross-sectional study from the International Children's Accelerometry Database (ICAD). Int J Obes. 2018;42(4):671-8.

9. Soininen P, Kangas AJ, Würtz P, Suna T, Ala-Korpela M. Quantitative serum nuclear magnetic resonance metabolomics in cardiovascular epidemiology and genetics. Circ Cardiovasc Genet. 2015;8(1):192-206.

10. Bell JA, Hamer M, Richmond RC, Timpson NJ, Carslake D, Smith GD. Associations of device-measured physical activity across adolescence with metabolic traits: prospective cohort study. PLoS Med. 2018;15(9):e1002649.

11. Kujala UM, Makinen V-P, Heinonen I, Soininen P, Kangas AJ, Leskinen TH, et al. Long-term leisure-time physical activity and serum metabolome. Circulation. 2013;127(3):340-8.

12. Jones PR, Rajalahti T, Resaland GK, Aadland E, Steene-Johannessen J, Anderssen SA, et al. Associations of physical activity and sedentary time with lipoprotein subclasses in Norwegian schoolchildren: The Active Smarter Kids (ASK) study. Atherosclerosis. 2019;288:186-93.

13. Resaland GK, Aadland E, Moe VF, Aadland KN, Skrede T, Stavnsbo M, et al. Effects of physical activity on schoolchildren's academic performance: The Active Smarter Kids (ASK) cluster-randomized controlled trial. Prev Med. 2016:91:322-8.

14. World Medical Association. World Medical Association Declaration of Helsinki: ethical principles for medical research involving human subjects. JAMA. 2013;310(20):2191-4.

15. Rich C, Geraci M, Griffiths L, Sera F, Dezateux C, Cortina-Borja M. Quality control methods in accelerometer data processing: defining minimum wear time. PLoS ONE. 2013;8(6):e67206.

16. Esliger DW, Copeland JL, Barnes JD, Tremblay MS. Standardizing and optimizing the use of accelerometer data for free-living physical activity monitoring. J Phys Act Health. 2005;2(3):366-83.

17. Evenson KR, Catellier DJ, Gill K, Ondrak KS, McMurray RG. Calibration of two objective measures of physical activity for children. J Sports Sci. 2008;26(14):1557-65.

18. Trost SG, Loprinzi PD, Moore R, Pfeiffer KA. Comparison of accelerometer cut points for predicting activity intensity in youth. Med Sci Sports Exerc. 2011;43(7):1360-8.

19. Okazaki M. Identification of unique lipoprotein subclasses for visceral obesity by component analysis of cholesterol profile in highperformance liquid chromatography. Arterioscler Thromb Vasc Biol. 2005;25(3):578-84.

20. Okazaki M, Yamashita S. Recent advances in analytical methods on lipoprotein subclasses: calculation of particle numbers from lipid levels by gel permeation HPLC using "spherical particle model". J Oleo Sci. 2016;65(4):265-82.

21. Kvalheim OM, Arneberg R, Grung B, Rajalahti T. Determination of optimum number of components in partial least squares regression from distributions of the root-mean-squared error obtained by Monte Carlo resampling. J Chemom. 2018;32(4):e2993.

22. Lin C, Rajalahti T, Mjøs SA, Kvalheim OM. Predictive associations between serum fatty acids and lipoproteins in healthy non-obese Norwegians: implications for cardiovascular health. Metabolomics. 2015;12(1):6.

23. Nanjee MN, Brinton EA. Very small apolipoprotein A-I-containing particles from human plasma: isolation and quantification by high-performance size-exclusion chromatography. Clin Chem. 2000;46(2):207-23.

24. Nordestgaard BG. A Test in context: lipid profile, fasting versus nonfasting. J Am Coll Cardiol. 2017;70(13):1637-46.

25. Cole TJ, Lobstein T. Extended international (IOTF) body mass index cutoffs for thinness, overweight and obesity. Pediatr Obes. 2012;7(4):284-94.

26. Carel J-C, Léger J. Precocious puberty. N Engl J Med. 2008;358(22):12. 
27. Gao X, Starmer J, Martin ER. A multiple testing correction method for genetic association studies using correlated single nucleotide polymorphisms. Genet Epidemiol. 2008;32(4):361-9.

28. Ferreira DLS, Williams DM, Kangas AJ, Soininen P, Ala-Korpela M, Smith $\mathrm{GD}$, et al. Association of pre-pregnancy body mass index with offspring metabolic profile: analyses of 3 European prospective birth cohorts. PLoS Med. 2017;14(8):e1002376.

29. Kujala UM, Vaara JP, Kainulainen H, Vasankari T, Vaara E, Kyröläinen H. Associations of aerobic fitness and maximal muscular strength with metabolites in young men. JAMA Netw Open. 2019;2(8):e198265.

30. Sniderman AD, Couture P, Martin SS, DeGraaf J, Lawler PR, Cromwell WC et al. Hypertriglyceridemia and cardiovascular risk: a cautionary note about metabolic confounding. J Lipid Res. 2018;59(7):1266-75.

31. Ference BA, Kastelein JJP, Ginsberg HN, Chapman MJ, Nicholls SJ, Ray KK, et al. Association of genetic variants related to CETP inhibitors and statins with lipoprotein levels and cardiovascular risk. JAMA. 2017;318(10):947-56.

32. Borén J, Chapman MJ, Krauss RM, Packard CJ, Bentzon JF, Binder CJ, et al. Low-density lipoproteins cause atherosclerotic cardiovascular disease: pathophysiological, genetic, and therapeutic insights: a consensus statement from the European Atherosclerosis Society Consensus Panel. Eur Heart J. 2020:41(24):2313-30.

33. Richardson TG, Sanderson E, Palmer TM, Ala-Korpela M, Ference BA, Smith $\mathrm{GD}$, et al. Evaluating the relationship between circulating lipoprotein lipids and apolipoproteins with risk of coronary heart disease: a multivariable Mendelian randomisation analysis. PLoS Med. 2020;17(3):e1003062.

34. Ala-Korpela M. The culprit is the carrier, not the loads: cholesterol, triglycerides and apolipoprotein B in atherosclerosis and coronary heart disease. Int J Epidemiol. 2019;48(5):1389-92.

35. Chait A, Ginsberg HN, Vaisar T, Heinecke JW, Goldberg IJ, Bornfeldt KE. Remnants of the triglyceride-rich lipoproteins, diabetes, and cardiovascular disease. Diabetes. 2020;69(4):508-16.

36. Borén J, Williams KJ. The central role of arterial retention of cholesterolrich apolipoprotein-B-containing lipoproteins in the pathogenesis of atherosclerosis: a triumph of simplicity. Curr Opin Lipidol. 2016:27(5):473-83.

37. Carson V, Tremblay MS, Chaput J-P, McGregor D, Chastin S. Compositional analyses of the associations between sedentary time, different intensities of physical activity, and cardiometabolic biomarkers among children and youth from the United States. PLoS ONE. 2019;14(7):e0220009.

38. Sondergaard E, Rahbek I, Sørensen LP, Christiansen JS, Gormsen LC, Jensen MD, et al. Effects of exercise on VLDL-triglyceride oxidation and turnover. Am J Physiol-Endocrinol Metab. 2011;300(5):E939-44.

39. Magkos F, Wright DC, Patterson BW, Mohammed BS, Mittendorfer B. Lipid metabolism response to a single, prolonged bout of endurance exercise in healthy young men. Am J Physiol-Endocrinol Metab. 2006;290(2):E355-62.

40. Herd SL, Lawrence JEM, Malkova D, Murphy MH, Mastana S, Hardman AE. Postprandial lipemia in young men and women of contrasting training status. J Appl Physiol. 2000;89(5):2049-56.

41. Camont L, Chapman MJ, Kontush A. Biological activities of HDL subpopulations and their relevance to cardiovascular disease. Trends Mol Med. 2011;17(10):594-603.

42. MärzW, Kleber ME, Scharnagl H, Speer T, Zewinger S, Ritsch A, et al. HDL cholesterol: reappraisal of its clinical relevance. Clin Res Cardiol. 2017;106(9):663-75.

43. Lincoff AM, Nicholls SJ, Riesmeyer JS, Barter PJ, Brewer HB, Fox KAA, et al. Evacetrapib and cardiovascular outcomes in high-risk vascular disease. N Engl J Med. 2017;376(20):1933-42.

44. Holmes MV, Asselbergs FW, Palmer TM, Drenos F, Lanktree MB, Nelson CP, et al. Mendelian randomization of blood lipids for coronary heart disease. Eur Heart J. 2014;36(9):539-50.

45. Voight BF, Peloso GM, Orho-Melander M, Frikke-Schmidt R, Barbalic M, Jensen MK, et al. Plasma HDL cholesterol and risk of myocardial infarction: a mendelian randomisation study. Lancet. 2012;380(9841):572-80.

46. Ruiz-Ramie JJ, Barber JL, Sarzynski MA. Effects of exercise on HDL functionality. Curr Opin Lipidol. 2019;30(1):16-23.

47. Allard-Ratick MP, Kindya BR, Khambhati J, Engels MC, Sandesara PB, Rosenson RS, et al. HDL: fact, fiction, or function? HDL cholesterol and cardiovascular risk. Eur J Prev Cardiol. 2019;2047487319848214.

48. Jomard A, Osto E. High density lipoproteins: metabolism, function, and therapeutic potential. Front Cardiovasc Med. 2020;7:39
49. Würtz P, Wang Q, Kangas AJ, Richmond RC, Skarp J, Tiainen M, et al. Metabolic signatures of adiposity in young adults: mendelian randomization analysis and effects of weight change. PLoS Med. 2014;11(12):e1001765 Sheehan NA, editor.

50. Richmond RC, Davey Smith G, Ness AR, den Hoed M, McMahon G, Timpson NJ. Assessing causality in the association between child adiposity and physical activity levels: a mendelian randomization analysis. PLoS Med. 2014;11(3):e1001618.

51. Mattocks C, Leary S, Ness A, Deere K, Saunders J, Kirkby J, et al. Intraindividual variation of objectively measured physical activity in children. Med Sci Sports Exerc. 2007;39(4):622-9.

52. Peddie MC, Rehrer NJ, Perry TL. Physical activity and postprandial lipidemia: Are energy expenditure and lipoprotein lipase activity the real modulators of the positive effect? Prog Lipid Res. 2012;51(1):11-22.

53. Guthold R, Stevens GA, Riley LM, Bull FC. Global trends in insufficient physical activity among adolescents: a pooled analysis of 298 populationbased surveys with 1.6 million participants. Lancet Child Adolesc Health. 2020:4(1):23-35.

\section{Publisher's Note}

Springer Nature remains neutral with regard to jurisdictional claims in published maps and institutional affiliations.
Ready to submit your research? Choose BMC and benefit from:

- fast, convenient online submission

- thorough peer review by experienced researchers in your field

- rapid publication on acceptance

- support for research data, including large and complex data types

- gold Open Access which fosters wider collaboration and increased citations

- maximum visibility for your research: over $100 \mathrm{M}$ website views per year

At BMC, research is always in progress.

Learn more biomedcentral.com/submissions 